CULTURA, LENGUAJE Y REPRESENTACIÓN / CULTURE, LANGUAGE AND REPRESENTATION · ISSN 1697-7750 • VOL. XV \2016, PP. 23-33 REVISTA DE ESTUDIOS CULTURALES DE LA UNIVERSITAT JAUME I / CULTURAL STUDIES JOURNAL OF UNIVERSITAT JAUME I

DOI: HTTP://DX.DOI.ORG/10.6035/CLR.2016.15.2

\title{
Reintroduciendo la dimensión política en el discurso del desarrollo humano: Una lectura crítica del enfoque de las capacidades
}

\author{
Bringing politics back into the human development discourse: \\ a critical insight of the capability approach
}

GUILLERMO OTANO JIMÉNEZ

UNIVERSIDAD PÚBLICA DE NAVARRA

Recibido: 24-10-2014

Aceptado: 11-02-2015

RESUMEN: El objetivo de este artículo es explorar la relación entre el enfoque de las capacidades y el discurso del desarrollo humano. El argumento que sostengo es que, para convertirse en una práctica social, el discurso del desarrollo humano debe incorporar la idea de responsabilidad en el diagnóstico de las desigualdades. En esta línea, la controversia entre los dos principales precursores del enfoque, el economista indio Amartya Sen y la filósofa americana Martha Nussbaum, ha contribuido a esclarecer el significado y el alcance de las políticas públicas (public policy) orientadas al desarrollo humano. Sin embargo, su defensa de la razón pública como mecanismo de cambio social carece de un análisis sistemático de las relaciones de poder. Para atender a la dimensión política (en el sentido que los anglosajones dan al término «politics») del discurso del desarrollo humano, propongo introducir el concepto de «empoderamiento comunicativo» como capacidad colectiva. Dicho concepto sirve para describir la articulación entre tres tipos de prácticas sociales relacionadas con el desarrollo humano: 1) la toma de conciencia de las desigualdades; 2) la movilización de recursos para la acción colectiva; y 3 ) la participación en la definición pública de los «problemas sociales». Estos tres tipos de prácticas van encaminadas al fortalecimiento del compromiso democrático con el bienestar humano y la justicia social.

Palabras clave: discurso, cambio social, capacidades, desarrollo humano, justicia social.

ABSTRACT: The aim of this paper is to explore the relationship between the capability approach and the human development discourse. My argument is that, in order to become a social practice, the human development discourse should introduce the concept 
of responsibility in the assessment of inequalities. Along these lines, the controversy between the Indian economist Amartya Sen and the American philosopher Martha Nussbaum has enriched our understanding of the public policies that suit the aims of human development best. However, their defense of public reason as a mechanism of social change seems to be insufficient because it does not pay attention to power relationships. This is one of the main weaknesses of the human development paradigm, and the only way to deal with it is to bring politics back into the human development discourse. In order to do so, I will propose the concept of «communicative empowerment» as a collective capability. This concept is designed to articulate three kinds of social practices: 1) the rising of awareness concerning inequalities; 2) the resource mobilization in order to promote collective action; 3) the social participation of the people on the public definition of «social problems». Each of these practices is orientated to reinforce the public commitment with human well-being and social justice.

Keywords: discourse, social change, capabilities, human development, social justice.

\section{Introducción}

El interés suscitado por el enfoque de la(s) capacidad(es) no ha dejado de crecer en las últimas dos décadas, desde que el Programa de las Naciones Unidas para el Desarrollo (PNUD) lo tomase como referencia para presentar una forma alternativa de medir el progreso humano que terminase con la «tiranía del PIB» en el estudio del desarrollo. Uno de los ejes fundamentales de su trabajo consistió en elaborar un concepto medible de desarrollo humano que sirviese para hacer comparaciones de bienestar (entre países, regiones y grupos sociales) en función de una serie de capacidades básicas (relacionadas con la educación, la salud y el nivel de vida). En su favor hay que reconocer que el conocimiento que proporciona este tipo de comparaciones ha cambiado nuestra percepción del desarrollo. Hoy sabemos que el crecimiento económico es una condición necesaria, pero no suficiente para que mejore la calidad de vida de las personas. Para conseguir esto último es necesario promover el desarrollo humano activamente o, como suele decirse en la jerga de la cooperación internacional, empoderar a la gente. Ahora bien, en un contexto como el que nos presenta la globalización neoliberal, en el que la ciudadanía de los países desarrollados asiste impotente al desmantelamiento del estado del bienestar y el consiguiente crecimiento de las desigualdades internas; en el que las potencias emergentes, siguiendo la fórmula desarrollista, anteponen en muchos casos el 
crecimiento económico a la democracia y el bienestar humano; y los países «en vías de desarrollo» carecen de poder para negociar acuerdos comerciales justos, ¿qué entendemos por empoderar? ¿Es el desarrollo humano un mero discurso sin conexión con la realidad concreta o, por el contrario, es un discurso conectado a una praxis?

El argumento que voy a sostener apunta en esta última dirección. Considero, al igual que otros autores críticos con el enfoque de las capacidades, que la praxis del desarrollo humano ha de consistir en "construir instituciones más justas» (Deneulin, 2006; 2014: 45 y ss.). Tanto Sen como Nussbaum, los principales precursores del enfoque en el ámbito académico, han trabajado esta dimensión relativa a la justicia en sus textos más recientes, aunque mantengan discrepancias al respecto. Analizaré las posturas de ambos desde el punto de vista de mi argumento: y es que para convertirse en una praxis, el discurso del desarrollo humano ha de ir más allá de la mera evaluación de las desigualdades de capacidades e incluir alguna manera de atribuir responsabilidades en el seno de la comunidad política. Este propósito, a su vez, requiere introducir el análisis de las relaciones de poder en el estudio de los procesos de desarrollo humano.

\section{El enfoque de la capacidad según Amartya Sen}

A finales de los años setenta, en una célebre conferencia que reunió a expertos en economía del bienestar, entre los cuales se encontraba Sen con destacados teóricos de la justicia, como el filósofo John Rawls, el economista indio planteó una pregunta que durante las décadas siguientes serviría de base para reconciliar la economía y la ética en torno a la noción de desarrollo humano. La pregunta en cuestión, que terminó dando título a su intervención, era: «Igualdad, ¿de qué?». El argumento que esgrimió entonces es que todas las teorías filosóficas sobre el bienestar y la justicia manifiestan de un modo u otro un interés por la igualdad. Pero este consenso tácito en reconocer la importancia de la igualdad se evapora, sin embargo, cuando nos preguntamos qué «variable focal» hemos de escoger para evaluar las desigualdades, de ahí su pregunta (Sen, 1980). En aquella ocasión, afirmó que el concepto intuitivo de capacidad humana ofrece un espacio más apropiado a la hora de evaluar el bienestar humano que la utilidad, los recursos, el ingreso o los bienes primarios de Rawls. Durante toda la década siguiente se dedicó a profundizar en su crítica de las debilidades teóricas que presentan estas corrientes, al tiempo que comenzaba a trabajar en los fundamentos de su propio enfoque: el enfoque de la capacidad (Sen, 1980; 1983; 1984; $1985 a ; 1985 b ; 1989)$. 
Para el economista indio, a la hora de juzgar cómo de bien se encuentra una persona, hemos de evitar tanto el «subjetivismo extremo» de las opiniones personales acerca del bienestar propio, error que comete la economía utilitarista del bienestar, como el «fetichismo de las mercancías» (Marx, 1887: 41-55; citado en Sen, 1989: 44) en el que incurren las principales interpretaciones de la justicia distributiva. Resumiendo: más allá de lo aceptable, en su argumentación podría decirse que el subjetivismo no permite detectar las injusticias, entre otras cosas, porque las personas pueden declarar que son felices incluso viviendo en condiciones de vida miserables; mientras que las teorías de la justicia distributiva que defienden la igualdad de oportunidades, aun apuntando en la buena dirección, erran en su concepción convencional de las «oportunidades», pues a menudo éstas se identifican con la posesión de ingresos, recursos o bienes primarios (Rawls 1971 y 2012: sección 17.2), y no con lo que las personas pueden hacer o ser a partir de los medios que tienen a su alcance. Si lo que realmente nos preocupa es esto último (es decir, la igualdad real de oportunidades), la información que hemos de utilizar a la hora de hacer juicios morales es aquella que atañe a lo que las personas son capaces de ser o hacer con sus vidas.

Para arrojar luz sobre dicha cuestión, Sen propone fijar la atención en dos conceptos interrelacionados. El primero de ellos, de raigambre aristotélica, es el de «funciones» o «funcionamientos» humanos, y hace referencia a «las diversas cosas que una persona puede valorar hacer o ser» (Sen, 1992 y $1999 a$ : 75). Desde esta perspectiva, la vida humana se puede interpretar en clave de funcionamientos, pues éstos constituyen los estados y acciones que la persona logra realizar con éxito. Los funcionamientos valorados pueden cubrir desde los aspectos más elementales para la supervivencia (como estar bien nutrido o evitar enfermedades evitables) hasta los más complejos (como la participación social o el autorrespeto).

El concepto de capacidades es más general, y deriva de la idea de funcionamientos. La capacidad hace referencia a un tipo de libertad: «la libertad fundamental para conseguir distintas combinaciones de funciones (o, en términos menos formales, la libertad para lograr diferentes estilos de vida)» (Sen, $1999 a$ : 75). De manera que al introducir las capacidades en la base informativa del enfoque, Sen reconoce el valor intrínseco de la libertad, pues en muchas ocasiones tenemos razones para valorar el hecho de elegir por encima de los efectos que la elección tenga en nuestro bienestar. De este modo, Sen rompe con la imagen del beneficiario pasivo que inconscientemente se asocia a las políticas públicas, para reivindicar la «condición de ser agente» que todas las personas tenemos a la hora de decidir cómo queremos vivir nuestra vida. 
Desde finales de los noventa, Sen ha hecho hincapié en que el desarrollo, entendido como libertad, ha de consistir en la «expansión de las capacidades que tenemos razones para valorar» (Sen, $1999 a$; Shaik, 2006). A menudo, como en el caso del PNUD, esta expresión se ha asociado a una serie de capacidades básicas (p. ej., la capacidad para llevar una vida larga y saludable, ser capaz de leer y escribir, la capacidad para aparecer en público sin avergonzarse de uno mismo, la capacidad para participar en la vida social, etc.). Sin embargo, Sen se ha mostrado reticente a elaborar una lista cerrada de capacidades, alegando que es preferible dejarla abierta y que sean los propios implicados en el contexto de la evaluación quienes decidan libremente -haciendo uso del escrutinio razonado y la deliberación pública- cuáles son aquellas capacidades que han de promoverse mediante la acción pública. Merece la pena examinar la controversia que ha mantenido con la filósofa Martha Nussbaum respecto a esta cuestión.

\section{La controversia con Nussbaum}

Los primeros trabajos de Nussbaum sobre la idea de las capacidades también se remontan a la década de los ochenta y, al igual que Sen, desde entonces ha venido trabajando la fundamentación teórica del enfoque (Nussbaum, 1988a; $1988 b ; 1992 ; 2000 ; 2002 a ; 2002 b ; 2007 ; 2011 a$ y 2012). De hecho, entre los años 1987 y 1989, colaboraron juntos para el instituto WIDER y el fruto de aquel trabajo quedó plasmado en algunas publicaciones (Nussbaum y Sen, 1989 y 1993). Ambos coinciden en señalar que el espacio de las capacidades es el más idóneo para teorizar sobre el bienestar y la justicia. Sin embargo, difieren en su manera de entender la construcción de instituciones más justas: el razonamiento de Sen es inductivo, es decir, evita explicitar un criterio de justicia para que sean las propias personas quienes definan, a través del debate público, qué situaciones pueden considerarse más o menos injustas y cuáles son las capacidades que deberían fortalecerse en cada caso para paliar dichas injusticias (Sen 2009); mientras que el de Nussbaum es deductivo, puesto que infiere, a partir de la noción de dignidad humana, una lista de diez capacidades humanas centrales que, a su juicio, deberían universalizar las instituciones de toda sociedad que pretenda ser justa. En ella se incluyen capacidades relacionadas con el logro de una vida larga y saludable, la salud y la integridad corporal, los sentidos, la imaginación y las emociones, el ejercicio de la razón práctica, la afiliación, el respeto por la naturaleza, el ocio y el control material y político de nuestro entorno. El objetivo de Nussbaum es que las dimensiones inclui- 
das en dicha lista sean instituidas constitucionalmente para que la ciudadanía pueda reclamar al Estado su responsabilidad en relación con la protección y el fomento de las mismas.

La aproximación de Nussbaum se acerca a mi propuesta para «repolitizar» el enfoque de las capacidades en la medida en que incluye una interpretación de las capacidades como derechos fundamentales que sirve como principio de atribución de responsabilidades en el marco del Estado. La lectura de Sen apunta en una dirección similar y admite igualmente que las capacidades elementales -aun siendo el de «capacidad» un concepto más general- pueden entenderse como derechos humanos y, por lo tanto, fundamentar reclamaciones en las instancias que correspondan (sean un tribunal supremo o las cortes de justicia internacionales) (Sen, 2004). Sin embargo, el economista indio ha mostrado en diversas ocasiones su desacuerdo con el proceder de Nussbaum, no tanto por rechazar la formulación de una lista de capacidades, sino por la insistencia en una lista canónica de capacidades predeterminadas (Sen, 2004: 77 y 2005:157). A un proyecto de ese tipo -que, en el fondo, es similar al planteado por Nussbaum-se le pueden hacer, según Sen, dos objeciones de tipo metodológico y una de tipo ético.

La primera es que el contenido de la lista no debería ser independiente del contexto donde se produce la evaluación y sus objetivos. No es lo mismo evaluar las desigualdades existentes en el sistema educativo español que la pobreza en los países «en desarrollo», por ejemplo. Cada objeto de estudio requerirá una lista de capacidades ad hoc, que parta de la realidad concreta que se investiga. La segunda razón es que una vez fijado el objeto de estudio, habría que decidir el peso específico y la importancia de cada una de ellas, y no deberíamos prefijar esta cuestión, dadas las cambiantes condiciones sociales y la necesidad de adaptar nuestra evaluación a las mismas (p. ej., aparición de nuevos factores de exclusión, etc.). Y la tercera es que al cerrar la lista y poner el énfasis en la responsabilidad gubernamental para su cumplimiento, podemos terminar justificando una forma de «paternalismo» desmovilizadora. Estas tres críticas me parecen suficientemente razonables para afirmar que el enfoque de Sen es más versátil y dinámico a la hora de plantear cambios sociales en diferentes escalas. De hecho, Nussbaum las ha aceptado parcialmente, y en sus últimos escritos pone un énfasis mayor al afirmar que su lista está abierta al debate, que es igualmente incompleta, y que los umbrales de capacidad mínimos han de establecerse a través del diálogo público. Sin embargo, hay una crítica que podría ser extensible a ambos planteamientos y es que ninguno de los dos autores incorpora un análisis sistemático de las relaciones de poder que distorsionan en la práctica al funcionamiento de la democracia. 


\section{Introduciendo las relaciones de poder en el razonamiento público}

En la literatura sobre desarrollo humano es común afirmar que la calidad de una democracia depende del compromiso que muestran las élites políticas, y la ciudadanía en general, con el bien común. Por tal suele entenderse el respeto a las libertades civiles y políticas (p. ej., libertad de culto, de expresión, de conciencia...) y el empoderamiento ciudadano a través de la provisión de una serie de servicios sociales básicos (sanidad, educación, etc.) que se conciben generalmente como derechos económicos. Ambas cosas suelen aparecer en los textos constitucionales de las democracias liberales como derechos fundamentales, puesto que permiten a la gente vivir la vida con un mínimo de dignidad y tener la oportunidad de perseguir su concepción particular del bien. Evidentemente, la forma institucional que adoptan estas demandas varía en función de la historia y la cultura política de cada país. En general, la historia de la democracia moderna es la historia de las luchas por delimitar las fronteras de la comunidad política y definir el contenido de lo que entendemos por ciudadanía. Los derechos reconocidos por el Estado a sus ciudadanos, en este sentido, han sido reformulados en diferentes momentos (junto con sus correspondientes deberes) para ir incluyendo a quienes históricamente se les había negado la condición de ciudadanos. La lucha contra la esclavitud durante el siglo XVIII, el auge del movimiento obrero y el sufragismo desde finales del siglo XIX, los movimientos por los derechos civiles y la emancipación de las mujeres en la segunda mitad del xx o la reivindicación de los derechos indígenas que presenciamos a comienzos de este siglo XXI, constituyen diversas expresiones del movimiento hacia la autonomía que caracteriza el despliegue institucional de la modernidad política (Wagner, 2008: capítulos 2-4).

En sus escritos más recientes, Sen defiende -como también lo hará Nussbaum- una concepción de la democracia que va más allá de la representación obtenida en las urnas, para centrarse en el ejercicio de la razón pública o la deliberación colectiva (Sen, 1999 b). Según el economista indio, sólo cuando las instituciones de la democracia representativa se complementan con una cultura política democrática se fortalece verdaderamente el desarrollo humano (véase también Inglehart y Welzel, 2005), y aporta tres razones fundamentales para defender dicha afinidad (Sen, $1999 a$ : 151 y ss.). La primera de ellas es que la democracia reconoce el valor intrínseco de la libertad humana para elegir el tipo de vida que apreciamos. La segunda es que, además, cumple un rol instrumental a la hora de promover el progreso humano, puesto que permite a los individuos expresar sus demandas en público y esto constituye un poderoso incentivo político para que los gobernantes se preocupen de atenderlas. Es, de hecho, una de las 
razones por las cuales ninguna de las grandes hambrunas del siglo XX se produjo en países democráticos (Sen, 1981). En tercer lugar, pero no menos importante, el escrutinio razonado de nuestros valores y nuestras formas de vida ejerce una función constructiva a la hora de definir nuestras necesidades económicas. Por ejemplo, permite modificar nuestra comprensión del bienestar humano, e incluso abre la puerta al cuestionamiento de nuestros modelos de consumo, etc.

Ahora bien, nada de esto puede hacernos perder de vista que muchas de las democracias existentes están atravesadas por desigualdades económicas que a menudo están relacionadas con otras muchas formas de discriminación (en función del género, la etnia o la religión). En su última obra publicada hasta la fecha, junto con Jean Drèze, Sen examina esta cuestión en el contexto de la India (Drèze y Sen, 2013). Sus análisis muestran que los ingentes beneficios del crecimiento económico indio durante la última década no se han repartido como cabría esperarse, a pesar de que la India es un país democrático desde que obtuviera la independencia en 1947. La razón, como adelanta en las páginas introductorias, es que «la democracia no consiste sólo en la política electoral y las libertades civiles, sino también en la equitativa distribución del poder» (Sen y Drèze, 2013: XI). La atención que se presta a las demandas provenientes de los grupos más desfavorecidos depende en buena medida de su capacidad colectiva para participar en la definición de los problemas sociales.

El logro de este tipo de capacidad colectiva se corresponde con lo que he denominado como empoderamiento comunicativo y ha de entenderse como un proceso que se articula en torno a tres ejes: 1) la toma de conciencia de las injusticias presentes en una situación para determinado colectivo, que podría fomentarse mediante la aplicación participativa del enfoque de las capacidades a la hora de definir sus demandas e identificar las estructuras sociales que reproducen las injusticias; 2) la movilización de recursos, tanto tangibles (como materiales didácticos, dinero para campañas, acceso a internet, etc.) como intangibles (conocimiento de legislación, redes sociales, etc.); y 3) la puesta en escena, que puede ir dirigida a facilitar la participación política directa (mediante espacios consultivos, alianzas políticas, movilización del voto) o indirecta (a través de medios de comunicación, nuevas tecnologías, etc.), con vistas a hacer llegar sus voces a la esfera pública y establecer redes de solidaridad. Estas tres cuestiones son necesarias para que el ejercicio de la responsabilidad que mencioné más arriba se convierta en una praxis real y no en una mera declaración de principios. 


\section{Conclusiones}

El principal hallazgo de esta comunicación pasa por reconocer las limitaciones del enfoque de las capacidades a la hora de traducirse en prácticas sociales. El problema está en que su marco teórico se ha construido a caballo entre, por un lado, la teoría de la elección social y la economía del bienestar (Sen) y, por otro, la filosofía política (Nussbaum); y en ambos casos se trata de disciplinas y corrientes teóricas que no han prestado suficiente atención a los aspectos relacionales del desarrollo humano y, especialmente, a las relaciones de poder (Otano Jiménez, 2015). Es por ello que, en última instancia, independientemente de la postura que se adopte en relación con la lista, su defensa de la democracia y la razón pública como mecanismos de cambio social aparece en muchos de sus textos como un ideal más que como una praxis real, con todas las imperfecciones, dilemas y demás inconvenientes que se puedan asociar a este adjetivo.

La noción de empoderamiento comunicativo que he propuesto escuetamente está pensada para que las organizaciones sociales que trabajan con los colectivos más desfavorecidos puedan resignificar su labor desde el discurso del desarrollo humano. Este movimiento no es un capricho personal ni una maniobra para cooptar sus acciones dentro de un discurso que, si bien antaño era alternativo, hoy va camino de convertirse en dominante. Es simplemente una manera de tender puentes entre la red de académicos e investigadores que se formó a comienzos de esta década para profundizar en las aplicaciones del enfoque de las capacidades (la Human Capability and Development Association) y los activistas y organizaciones que trabajan por lograr un mundo más justo. La cercanía entre la reflexión teórica y el aprendizaje práctico de la experiencia que tienen estas organizaciones y movimientos no puede ser más que beneficiosa en ambas direcciones, tanto para lograr una academia que vuelva su mirada sobre los problemas reales de la gente, como para encontrarles soluciones duraderas en el tiempo.

\section{Referencias bibliográficas}

Deneulin, S. (2014): Wellbeing, Justice and Development Ethics, Nueva York, Routledge.

- (2006): The capability approach and the praxis of development, Houndmills, Palgrave Macmillan.

Drèze, J.; A. Sen (2013): An Uncertain Glory. India and its contradictions, Princeton (New Jersey), Princeton University Press. 
IngLeHART, R.; C. Welzel (2005): Modernización, cambio cultural y democracia: la secuencia del desarrollo humano, Madrid, Siglo XXI y CIS.

Nussbaum, M.; A. Sen (1989): «Internal Criticism and Indian Rationalist Traditions», en Krausz, M. (comp.): Relativism: Interpretation and Confrontation, Notre Dame (Indiana), University of Notre Dame Press, 229-325.

- (eds.) (1993): The Quality of Life, Nueva York, Oxford University Press.

NussbauM, M. (1988 a): «Nature, Function and Capability: Aristotle on Political Distribution», Oxford Studies in Ancient Philosophy, 1 supl.: 145-85.

- (1988 b): «Non-Relative Virtues: An Aristotelian Aroach», Midwest Studies in Philosophy, 13: 32-53.

- (1992): «Human Functioning and Social Justice: In Defense of Aristotelian Essentialism», Political Theory, 20: 202-246.

- (2000): «Aristotle, Politics, and Human Capabilities: A Response to Anthony, Arneson, Charlesworth, and Mulgan», Ethics, 3: 102-140.

- (2002 a): «Capabilities and Human Rights», en De Greiff, P.; C. Cronin, (comps.): Global Justice, Transnational Politics, Cambridge (Massachusetts), MIT Press, 117-150.

- (2002 b): Las mujeres y el desarrollo humano, Barcelona, Herder.

- (2007): Las fronteras de la justicia: consideraciones sobre la exclusión, Barcelona, Paidós.

- (2011): «Capabilities, Entitlements, Rights: Supplementation and Critique», Journal of Human Development and Capabilities: A Multi-Disciplinary Journal for People-Centered Development, 12 (1): 23-37.

- (2012): Crear capacidades. Propuesta para el desarrollo humano, Barcelona, Paidós.

Otano Jiménez, G. (2015): «La libertad como relación social: una interpretación sociológica del enfoque de las capacidades de Amartya Sen», Revista Iberoamericana de Estudios de Desarrollo, 4 (1): 98-127.

Pereira, G. (2004): «Autonomía, preferencias adaptativas y políticas públicas», Sistema, 178: 71-85.

RAwls, J. (1971): A Theory of Justice, Cambridge (Massachusetts), Harvard University Press.

RAwls, J. (2012): La justicia como equidad. Una reformulación, Barcelona, Paidós [1 ${ }^{\mathrm{a}}$ ed. en castellano: 2002].

ShaikH, N. (2006): «Desarrollo como libertad. Entrevista con Amartya Sen», Cuadernos del CENDES, sept.-dic., 23 (63): 123-137. Recuperado de http:// www.redalyc.org/articulo.oa?id=40306305

SEN, A. (1980): «Equality of what?», en McMurrin, S. (ed.): The Tanner Lectures on Human Values, Cambridge, Cambridge University Press. 
- (1981): Poverty and Famines. An essay on Entitlement and Deprivation, Oxford, Clarendon Press.

- (1983): «Development, Which way now?», The Economic Journal, 93 (372): 745-762.

- (1984): «Rights and Capabilities», en Sen, A. (ed.): Values and Development, Cambridge (Massachusetts), Harvard University Press, 307-324.

- (1985 a): Commodities and Capabilities, Amsterdam, Elsevier Science Publishers.

— (1985 b): «Well-being, Agency and Freedom: The Dewey Lectures 1984», The Journal of Philosophy, 82 (4): 169-221.

- (1989): «Development as Capability Expansion», Journal of Development Planning, 19: 41-58.

- (1992): Inequality Reexamined, Nueva York, Oxford University Press.

- (1999 a): Development as Freedom, Nueva York, Knopf.

- (1999 b): «Democracy as Universal Value», Journal of Democracy, 10 (3): 3-17.

- (2004): «Capacities, Lists and Public Reason: Continuing the Conversation», Feminist Economics, 10 (3): 77-80.

- (2005): «Human Rights and Capabilities», Journal of Human Development, 6 (2): 151-166.

- (2009): The Idea of Justice, Cambridge (Massachusetts), Harvard University Press.

WAGNer, P. (2008): Modernity as Experience and Interpretation. A New Sociology of Modernity, Cambridge, Polity Press. 